\title{
Conceptos actuales de medicina basada en la evidencia sobre el uso de fototerapia en psoriasis
}

\section{SEgUnda PARTE. Fototerapia CON LUZ ULTRAVIOLETA B}

\section{DE BANDA ESTRECHA EN PSORIASIS}

Current concepts of evidence-based medicine in phototherapy and photochemotherapy in psoriasis

Part II. Narrow band UVB in psoriasis

\section{Catalina Santa $^{1}$, Manuela Cadavid ${ }^{1}$, Sol Beatriz Jiménez ${ }^{2}$}

1. Médica, residente de Dermatología, Universidad CES, Medellín, Colombia

2. Médica dermatóloga; jefe, Servicio de Dermatología, Grupo Dermatología CES, Universidad CES, Medellín, Colombia

\section{Resumen}

La fototerapia tiene un papel inmunomodulador y por eso se usa para enfermedades inflamatorias de la piel. En la psoriasis se ha empleado la luz ultravioleta $B$ de banda ancha (BB-UVB), la luz ultravioleta B de banda estrecha (NB-UVB) y la luz ultravioleta A (UVA). La UVB de banda estrecha ha demostrado ser segura y eficaz y, hoy en día, se considera el tratamiento de primera línea para la psoriasis. Está indicada en las formas moderadas a graves y que ameritan un tratamiento complementario, además del tópico. Hay múltiples protocolos para su administración, dosis de inicio, incremento de la dosis en cada sesión, número de exposiciones a la semana, mantenimiento y dosis acumuladas. Se hace una revisión de los protocolos y la evidencia para el uso de ellos, de manera que el médico clínico pueda tomar decisiones adecuadas al momento de elegir un tratamiento con luz ultravioleta para la psoriasis.

PALABRAS CLAVE: fototerapia, psoriasis, NB-UVB

\section{Summary}

Phototherapy has immunomodulatory properties and this is why it is used for treating some inflammatory skin diseases. Broadband UVB (BB-UVB), narrow band UVB (NB-UVB) and UVA have been used for the treatment of psoriasis. NB-UVB has been shown to be effective and safe and nowadays it is considered the first line phototherapy in patients with psoriasis. It is considered for patients with psoriasis whom are not candidates for topical therapy alone. Multiple protocols for administering NB-UVB phototherapy have been used, including initial doses, percentage of dose increase after each session, number of doses recommended each week, maintenance regimes and maximum number of sessions. We reviewed the literature and different protocols published with the evidence showing their advantages and disadvantages so that the clinician can decide which regime is the best one for treating his/her patients.

KEY WORDS: phototherapy, psoriasis, NB-UVB

\section{Correspondencia:}

Catalina Santa

Email:

catalinasantavelez@yahoo.com

Recibido: 04 de octubre de 2012. Aceptado: 10 de diciembre de 2012.

No se reportan conflictos de intereses. 


\section{Introducción}

La fototerapia en forma de luz solar se ha usado por miles de años para el mejoramiento de diversos padecimientos cutáneos y, hoy en día, es uno de los pilares del tratamiento de la psoriasis ${ }^{1}$. Para la psoriasis, se ha utilizado en forma de fotoquimioterapia de luz ultravioleta A (UVA) más psoraleno (psoralen and ultraviolet A, PUVA), luz ultravioleta B (UVB) de banda ancha (broad band UVB, BB-UVB) y UVB de banda estrecha (narrow band UVB, NB-UVB) ${ }^{1}$. El papel que juega en el tratamiento de la psoriasis frente a otras modalidades terapéuticas aún no está claro, y algunas guías cuestionan su uso y prefieren emplear otros fármacos sistémicos o productos biológicos; sin embargo, todas las recomiendan en diferente medida ${ }^{2-7}$. La mayoría de las guías actuales consideran que la NB-UVB es la fototerapia de primera línea, ya que es más efectiva que la BB-UVB, se puede usar en pacientes con otras enfermedades concomitantes, en mujeres embarazadas y en niños, y su eficacia es comparable con la de la PUVA, sin su toxicidad ${ }^{8}$.

\section{Generalidades}

Las fuentes de UVB para fototerapia de cuerpo entero son principalmente lámparas fluorescentes, aunque también se pueden utilizar lámparas de halogenuros metálicos. Para fototerapia localizada, se usa el gas xenón o una fuente de luz filamentosa. Hay cuatro tipo de lámparas que emiten ondas de longitud dentro del espectro UVB: las lámparas de UVB de banda ancha, las de UVB de banda ancha selectivas (emiten ondas de una longitud menor de $290 \mathrm{~nm}$ en un porcentaje mucho menor que las primeras), las de UVB de banda estrecha (NB-UVB) que emiten el mayor porcentaje de ondas en el espectro de $311 \pm 2 \mathrm{~nm}$, y el láser excimer que emite ondas en el espectro de $308 \mathrm{~nm}$. También, hay lámparas de cloruro de xenón que emiten ondas de $308 \mathrm{~nm}^{9}$. Las lámparas de NB-UVB son las más efectivas para la psoriasis y el láser excimer se ha usado exitosamente para el tratamiento de placas localizadas 9 .

\section{Farmacodinamia}

La fototerapia con UVB ejerce sus efectos por medio de varios mecanismos. Su blanco principal es el ADN nuclear el cual absorbe los rayos de luz, generando dímeros de pirimidinas y otros fotoproductos que inhiben la síntesis de ADN; esto hace que el ciclo celular se detenga y se disminuya la proliferación de linfocitos y queratinocitos ${ }^{10}$. Además, aumenta la expresión de $p_{53}$, un gen supresor de tumores que regula el ciclo celular, causa apoptosis de linfocitos, disminuye la concentración de células de Langerhans y afecta la producción de citocinas por parte de las células inflamatorias ${ }^{1,11}$. La UVB también tiene efectos antimicrobianos al inducir la producción de algunos péptidos antimicrobianos por parte de los queratinocitos, ejerciendo un efecto antimicrobiano directo contra Staphylococcus aureus ${ }^{12,13}$.

\section{Efectividad}

El eritema que se desarrolla en la piel normal alrededor de la lesión es el factor limitante de la dosis de UVB que se puede administrar. Diferentes tipos de longitudes de onda producen diferente eritema9. Parrish y Jaenickeen demostraron en 1981 que la longitud de onda de $311 \mathrm{~nm}$ dentro del espectro UVB y que hace parte del espectro NB-UVB, era óptimo para el tratamiento de la psoriasis, ya que producía remisión sin eritema perceptible ${ }^{14}$, mientras que la UVB de banda ancha y la UVB selectiva requerían dosis mayores, incluso superiores a la dosis mínima de eritema9. Después de estos hallazgos, en múltiples estudios se demostró la superioridad de la NB-UVB sobre la BB-UVB, incluyendo algunos asignación aleatoria del lado derecho o izquierdo en un mismo individuo ${ }^{15-17}$. También, se demostró que la NB-UVB era equivalente o casi igual a la PUVA, sin la inconveniencia o toxicidad del psoraleno y el incremento en el riesgo de cáncer de piel que produce la fototerapia prolongada ${ }^{18-20}$.

\section{Seguridad}

La NB-UVB ha demostrado ser segura y puede utilizarse en pacientes con enfermedades concomitantes, en mujeres embarazadas y en niños ${ }^{21,22}$.

\section{Dosificación}

La dosificación de la NB-UVB se puede basar en la dosis mínima de eritema o en el fototipo de piel del paciente (según Fitzpatrick) por conveniencia del paciente y el médico ${ }^{1}$. La dosis mínima de eritema es la necesaria para producir eritema perceptible después de la administración de radiación ultravioleta. Debido a que la NBUVB produce remisión con dosis "suberitemogénicas", la dosis inicial puede ser 0,5 a 1,o de la dosis de eritema mínima (DEM), siendo o,7 DEM la más utilizada ${ }^{7,23-27}$.

La dosificación basada en la dosis mínima de eritema es la más segura para el paciente y presenta menor riesgo de quemadura. Se logra utilizando incrementos en una región no fotoexpuesta, como los glúteos, ya que las áreas fotoexpuestas presentan “engrosamiento” y se 
puede sobreestimar la dosis ${ }^{28}$. Para los fototipos I-III se inicia con $200 \mathrm{~mJ} / \mathrm{cm} 2$ y si no se alcanza a producir eritema se va aumentando $200 \mathrm{~mJ} / \mathrm{cm} 2$ cada sesión. Para los fototipos IV-VI se inicia con $600 \mathrm{~mJ} / \mathrm{cm} 2$ y también se aumentan $200 \mathrm{~mJ} / \mathrm{cm} 2$ de la dosis anterior progresivamente si no se produce eritema. La respuesta a cada dosis se debe leer a las 24 horas, ya que el eritema por NB-UVB tiene un pico a las 8-24 horas' ${ }^{29}$.

Kleinpenning, et al., llevaron a cabo un estudio doble ciego de asignación aleatoria en el 2009, en el cual se demostró que los esquemas con dosis altas eran más costo-efectivos y requerían menos sesiones de tratamiento que los de dosis bajas ${ }^{24}$. Algunos recomiendan incrementos de 10 a $20 \%$ de la DEM en cada sesión; otros usan incrementos altos (15 a $20 \%$ ) al inicio del tratamiento y, luego, incrementos de $10 \%$ en sesiones posteriores ${ }^{7,10}$. Algunos emplean aumentos de 30 a $40 \%$ por semana $^{30}$, pero los de 5 a $10 \%$ han sido efectivos ${ }^{25,27}$. Así por ejemplo, si la dosis mínima de eritema es $600 \mathrm{~mJ} /$ $\mathrm{cm}^{2}$, al usar el $50 \%$ de la DEM como dosis de inicio, se empezaría con $300 \mathrm{~mJ} / \mathrm{cm}^{2}$. Si luego se decide aumentar $10 \%$ de la DEM, la segunda dosis sería $(300+60)=360$ $\mathrm{mJ} / \mathrm{cm}^{2}$. La tercera dosis sería $(360+60)=420 \mathrm{~mJ} / \mathrm{cm}^{2} \mathrm{y}$, así, se continuaría el esquema ${ }^{7}$.

La mejor dosis para la fototerapia es aquella que es eficaz, que produce mínimos síntomas y no produce quemaduras, y que sí es lo suficientemente agresiva como para disminuir el número de sesiones ${ }^{9}$, por lo que se debe mantener un eritema leve durante el tratamiento $^{29}$. Los protocolos más citados para el uso de NBUVB en psoriasis, son los de la Sociedad Dermatológica Alemana y la de Zanolli. La Sociedad Dermatológica Alemana recomienda iniciar con o,7 DEM e incrementar la dosis total en $30 \%$ si no hay eritema, incrementar $20 \%$ si el eritema es leve, mantener la dosis si es moderado, y si es grave, se debe suspender el tratamiento hasta la remisión completa y reiniciar con $50 \%$ de la última dosis ${ }^{1}$. Zanolli recomienda iniciar con 0,5 DEM e incrementar $10 \%$ DEM en cada sesión.

Las recomendaciones de la Sociedad Dermatológica Alemana están basadas en más de 25 estudios de fototerapia y las recomendaciones de Zanolli se basan en opiniones de expertos ${ }^{1}$. La dosis de NB-UVB no debe sobrepasar cuatro a seis veces la DEM en las extremidades y dos a cuatro veces la DEM en el tronco'.

La determinación de la dosis mínima de eritema en cada paciente y el cálculo de la dosis que se debe administrar de acuerdo con esta, es la manera más efectiva y segura de dosificar la fototerapia ${ }^{1}$. Sin embargo, el cálculo basado en la dosis mínima de eritema es dispendioso y requiere de un mayor entrenamiento del personal que trabaja en los centros de fototerapia. Por esto, muchas veces se decide iniciar con dosis de acuerdo con el fototipo según Fitzpatrick. Zanolli y Feldman proponen que, si el fototipo es I o II, se inicie con dosis de $300 \mathrm{~mJ} / \mathrm{cm}^{2}$, si es III o IV, la dosis de inicio sea $500 \mathrm{~mJ} /$ $\mathrm{cm}^{2}$, y si es V o VI, la dosis inicial sea de $800 \mathrm{~mJ} / \mathrm{cm}^{2}{ }^{31,32}$.

Haddican, et al., compararon estas dosis de inicio propuestas por Zanolli, según el fototipo, con los protocolos de fototerapia de la Universidad de California en San Francisco (UCSF) y los protocolos propuestos por Morison. Los protocolos de la UCSF proponen iniciar con $130 \mathrm{~mJ} / \mathrm{cm}^{2}$ en pacientes con fototipo I, y Morison propone iniciar con $300 \mathrm{~mJ} / \mathrm{cm}^{2}$ en pacientes con fototipo I, igual que Zanolli. Las dosis iniciales van aumentando según el fototipo, de manera que los protocolos de la UCSF proponen una dosis de inicio de $400 \mathrm{~mJ} / \mathrm{cm}^{2}$, en pacientes con fototipo VI, y Morison propone una dosis de inicio de $1.500 \mathrm{~mJ} / \mathrm{cm} 2$, en pacientes con este fototipo, muy por encima de las propuestas por Zanolli33. Se evidencia una notoria diferencia en los protocolos y los autores de este artículo comparativo consideran que esto se debe a:

1. Mediciones infrecuentes de la irradiación de las cámaras.

2. Errores en la técnica para la medición de la irradiación.

3. Diferentes estándares de calibración para el fotómetro.

4. Defectos en el sistema de computarización para el cálculo de la dosis que se debe administrar ${ }^{33}$.

Los incrementos también se pueden hacer según el fototipo, incrementando $100 \mathrm{~mJ} / \mathrm{cm}^{2}$ cada tres días en los fototipos I a IV, o incrementando $150 \mathrm{~mJ} / \mathrm{cm}^{2}$ en los fototipos $\mathrm{V}$ y VI ${ }^{31,32}$. Si el paciente falta a la fototerapia una semana, puede continuar con la última dosis que recibió; si suspende una a dos semanas, se debe disminuir $25 \%$, si suspende dos a tres semanas, se disminuye $50 \%$, y si lo hace tres a cuatro semanas, se debe reini$\operatorname{ciar}^{31,32}$. Ver TABLA 1.

El número de sesiones por semana también puede variar; se ha demostrado que los esquemas de dos veces a la semana se demoran 1,5 veces más tiempo en lograr la remisión que los esquemas de tres veces por semana ${ }^{34}$, y no hay una diferencia estadísticamente significativa entre tres y cinco veces por semana ${ }^{35}$. Tres sesiones semanales minimizan el tiempo que se invierte en viajar a los centros de fototerapia y el tiempo fuera del trabajo y la familia, pero, menos de tres a la semana es inadecuado?.

El tratamiento se continúa hasta que no se observe mejoría clínica o hasta que haya remisión completa ${ }^{10}$; el número máximo de dosis se discute más adelante. Existe controversia en cuanto al mantenimiento a largo plazo, aunque hay cierta evidencia de que se puede lo- 


\section{Principios para tratamiento con NB-UVB en psoriasis}

Determinar la dosis inicial DEM según el tipo de piel según Fitzpatrick.

Iniciar con 50-70 \% de DEM tres a cuatro veces a la semana.

Aumentar la dosis 10 a $25 \%$ de la DEM en cada tratamiento o incrementar $100 \mathrm{~mJ} / \mathrm{cm}^{2}$ cada tres días en los fototipos I-IV o incrementar $150 \mathrm{~mJ} / \mathrm{cm}^{2}$ en los fototipos Vy VI.

Modificar la dosis según la respuesta clínica al tratamiento.

- Si no hay eritema: incrementar $30 \%$ la dosis total.

- Si el eritema es leve, apenas perceptible: incrementar $20 \%$ la dosis total

- Si el eritema es moderado: repetir la dosis hasta que disminuya el eritema y luego continuar incrementando la dosis.

- Si el eritema es grave: suspender la fototerapia hasta la mejoría clínica completa, luego iniciar con $50 \%$ de la última dosis y aumentar $10 \%$ cada sesión de ahí en adelante.

TABLA 1. Protocolos para NB-UVB (Sociedad Dermatológica Alemana - Zanolli) ${ }^{1,9,32}$

grar una remisión de la enfermedad más prolongada ${ }^{36}$. En caso de que se decida continuar con mantenimiento, este se hace después de alcanzar una remisión de $95 \%$ de las lesiones. Se hace una sesión semanal durante cuatro semanas con la dosis final a la que se escaló (M1), luego se disminuye $25 \%$ de la dosis final y se hacen cuatro sesiones (M2), una cada dos semanas. Finalmernte, se le aplican al paciente cuatro sesiones mensuales con $50 \%$ de la dosis máxima suministrada $\left(\mathrm{M}_{4}\right)^{32,37}$.

\section{Tratamiento combinado}

La fototerapia se puede combinar con algunas de las otras modalidades de tratamiento para alcanzar mejores resultados terapéuticos.

\section{Tratamientos tópicos más NB-UVB}

1. Análogos de la vitamina $D$. El calcipotrieno puede potenciar la fototerapia. Se debe administrar dos horas antes de la fototerapia o después de ella, ya que puede ser inactivado por la luz ultravioleta ${ }^{38}$.

2. Retinoides. El tazaroteno también potencia la fototerapia y disminuye el número de sesiones requeridas de $\mathrm{NB}-\mathrm{UVB}^{39,40}$, pero puede aumentar la irritación ${ }^{1}$.

3. Esteroides tópicos. No hay suficiente evidencia para recomendarlos ya que, a pesar de que pueden lograr una remisión más rápida, la combinación no muestra beneficios a largo plazo $0^{41-43}$.

4. Acido salicílico. El ácido salicílico empleado como queratolítico tiene propiedades fotoabsorbentes en el espectro UVB e, incluso, se ha utilizado en algunos fotoprotectores. Este no se debe aplicar antes de la fototerapia, debido a que la dosis que llega a la epidermis va a ser mucho menor o es impredecible y varía de una sesión a otra. Se puede administrar después de la fototerapia9.

5. Antralina y alquitrán de hulla. La combinación con antralina se recomienda. También, hay evidencia de que la combinación con alquitrán de hulla es efectiva ${ }^{1}$.

Antes de ingresar al paciente a la cámara de fototerapia se recomienda usar un agente tópico a base de petróleo, como puede ser el aceite mineral. Esto disminuye la interafase aire-queratina por la cual debe atravesar la luz ultravioleta antes de llegar al estrato granuloso y la epidermis más profunda 9 .

\section{Tratamientos sistémicos más NB-UVB}

1. Ciclosporina: no debe combinarse con la fototerapia por el aumento del riesgo de cáncer de piel, melanoma y no melanoma ${ }^{44}$.

2. Metotrexate: Hay que tener en cuenta que el metotrexato puede producir las llamadas reacciones de "recaída por quemadura solar" (sunburn recall reactions) y esta combinación se debe hacer durante la fase de remisión, ya que a largo plazo teóricamente puede incrementar el riesgo de cáncer de piel. También, se ha reportado recaída al suspender el metotrexato, por lo que se debe ser cauteloso o hacer un desmonte gradual ${ }^{45,46}$.

3. Acitretín. Hay evidencia de la combinación con acitretín, ya que este acelera la remisión y aumenta su porcentaje, por lo cual se le ahorran al paciente dosis acumuladas de radiación y se reducen los $\operatorname{costos}^{1}$. Se debe usar en psoriasis moderada a grave que no haya mejorado con NB-UVB, PUVA o acitretín solo, en pacientes con acceso limitado a la fototerapia o en quienes han fallado al metotrexate o la ciclosporina ${ }^{1}$. Antes de iniciar el retinoide, se deben solicitar pruebas de función hepática y perfil lipídico, y prueba de embarazo en mujeres en edad fértil. Luego, se inicia el acitretín 
a dosis bajas (0,3-0,5 $\mathrm{mg} / \mathrm{kg})$ cada día durante dos semanas; o, si el paciente ya venía recibiendo acitretín, se disminuye la dosis antes de iniciar la fototerapia. Después se inicia según el fototipo o 30 a $50 \%$ de la dosis mínima de eritema, incrementando 10 a $30 \%$ de la dosis si no hay eritema y o \% si hay eritema, o se omite la fototerapia en caso de que el eritema sea leve o haya dolor. En caso de iniciar el retinoide mientras el paciente está en fototerapia, se debe reducir $50 \%$ la dosis de radiación, manteniéndola por dos semanas. Es importante ser cauteloso con esta combinación, debido a que el acitretín induce adelgazamiento del estrato córneo y esto aumenta el riesgo de quemaduras $^{9,47}$.

4. Tratamientos biológicos. La combinación con productos biológicos puede ser beneficiosa, pero falta evidencia; hay estudios con alefacept, adalimumab y etanercept ${ }^{48-50}$.

\section{Contraindicaciones}

Hay pocas contraindicaciones absolutas. Estas incluyen xeroderma pigmentoso y lupus eritematoso sistémico ${ }^{51}$. Las contraindicaciones relativas son: antecedente de cáncer de piel, fotodaño, envejecimiento prematuro, reacciones fotoalérgicas, conjuntivitis o queratitis ${ }^{9,32}$.

\section{Efectos adversos}

\section{Agudos}

La fototerapia NB-UVB tiene el potencial de ocasionar quemadura cuando se emplea en dosis mayores a la DEM y el riesgo aumenta de manera proporcional con el número de milijulios acumulados. También, puede ocasionar ampollas en la piel en lesiones de psoriasis, por apoptosis en la placa psoriásica ${ }^{52}$. Se ha reportado exacerbación de las placas de dermatitis seborreica ${ }^{53}$.

\section{Crónicos}

Hasta el momento no se ha demostrado un aumento del riesgo de cáncer de piel con la fototerapia UVB ${ }^{54}$; sin embargo, esto puede deberse a periodos cortos de seguimiento y a que los tamaños de las muestras de los estudios no han sido adecuados.

Es difícil calcular una dosis tope debido a la falta de evidencia, pero además, depende del aumento del riesgo de cáncer de piel que el paciente está dispuesto a correr. Se han incorporado modelos matemáticos sobre incidencia de cáncer de piel no melanoma de acuerdo con la exposición solar y, según estos, se ha intentado definir el riesgo con el uso de UVB.

Diffey, et al., estimaron que ocho ciclos anuales de NBUVB, cada ciclo de 25 sesiones, incrementarían el riesgo de cáncer de piel no melanoma en 1,2 veces, en comparación con individuos que no recibieron dicho tratamiento ${ }^{55}$. Así, a los pacientes dispuestos a correr este aumento de $20 \%$ del riesgo, se les recomendaría una dosis tope de 200 sesiones si recibieron fototerapia sin cubrirse la cara. Sin embargo, el riesgo se modificaría dependiendo del tipo de piel, si la cara y los genitales fueron cubiertos y otros factores de predisposición, como los fototipos I-II (sujetos pelirrojos). Además, este modelo asume que la NB-UVB es igual de carcinogénica que la luz solar para la misma dosis mínima de eritema, pero este no es necesariamente el caso. La fototerapia NB-UVB puede ser el doble de carcinogénica que la luz solar (según algunos modelos animales) y, por ende, el tope de sesiones recomendadas sería la mitad 55 .

Recientemente, se publicó un estudio prospectivo de 30 años para analizar el riesgo de carcinoma escamocelular en pacientes que recibieron PUVA. Se hizo un análisis multivariado para calcular el riesgo relativo (RR) en pacientes que también recibieron UVB. Se encontró un RR de 0,85 a 1,15, con menos de 100 sesiones de UVB, de 1,32 a 1,68 , con 100 a 300 sesiones, y de 1,32 a 1,68, con más de 300 sesiones ${ }^{56}$.

\section{Fototerapia NB-UVB localizada}

El láser excimer emite rayos de $308 \mathrm{~nm}$ en el espectro NB-UVB. También, se puede utilizar una fuente de luz monocromática que emite rayos de $308 \mathrm{~nm}$ que no es láser o cámaras pequeñas de NB-UVB, con la ventaja de tratar solamente las placas de psoriasis, lo cual ahorra dosis de irradiación a la piel sana circundante y, por ende, pueden emplearse dosis mayores y se logra una remisión más rápida. Además, se ha demostrado que esto puede ser menos costoso que el tratamiento con productos biológicos o la fototerapia de cuerpo entero. Los efectos secundarios son similares a los mencionados anteriormente e incluyen eritema, ampollas, hiperpigmentación, erosiones, ardor y prurito. Esta se debe considerar en variantes localizada de psoriasis, como la forma pustulosa palmo-plantar ${ }^{57}$.

\section{Conclusión}

La fototerapia es un método ampliamente utilizado en el mundo desde hace más de 40 años y aún sigue siendo una buena alternativa terapéutica en los pacientes con psoriasis, dado su bajo costo. Puede combinarse con 
otros tratamientos, tanto tópicos como sistémicos, con buenos resultados. En la era de los tratamientos biológicos, la fototerapia mantiene su efectividad cuando se compara con algunos de ellos.

La NB-UVB es una alternativa muy eficaz y segura en el tratamiento de los pacientes con psoriasis. Los protocolos utilizados alrededor del mundo difieren en $\mathrm{mu}$ chos aspectos, aunque es claro que lo ideal es manejar la dosis según la DEM para individualizarlo. Las sesiones deben realizarse tres veces a la semana y aumentar la dosis según la respuesta clínica. Varios autores han propuesto que se empiecen a utilizar técnicas para estandarizar los esquemas de administración de la fototerapia, para que todos los pacientes se beneficien por igual y los estudios a nivel mundial puedan ser comparables. Sin embargo, esto es difícil ya que cada centro utiliza los protocolos que más se adapten a su población.

Esta revisión actualizada con medicina basada en la evidencia, le permitirá al médico clínico seleccionar el esquema terapéutico que se acomoda mejor a las necesidades de sus pacientes.

\section{Referencias}

5. Lapolla W, Yentzer BA, Bagel J, Halvorson CR, Feldman SR. A review of phototherapy protocols for psoriasis treatment. J Am Acad Dermatol. 2011;64:936-49.

6. Menter A, Korman NJ, Elmets CA, Feldman SR, Gelfand JM, Gordon KB, et al. Guidelines of care for the management of psoriasis and psoriatic arthritis. J Am Acad Dermatol. 2011;65:137-74.

7. Callen JP, Krueger GG, Lebwohl M, McBurney EI, Mease P, Menter $\mathrm{A}$, et al. AAD consensus statement on psoriasis therapies. J Am Acad Dermatol. 2003;49:897-9.

8. Guenther L, Langley RG, Shear NH, Bissonnette R, Ho V, Lynde C, et al. Integrating biologic agents into management of moderateto-severe psoriasis: A consensus of the Canadian Psoriasis Expert Panel. J Cutan Med Surg. 2004;8:321-37.

9. Sterry W, Barker J, Boehncke W-H, Bos JD, Chimenti S, Christophers $\mathrm{E}$, et al. Biological therapies in the systemic management of psoriasis: International Consensus Conference. Br J Dermatol. 2004;151(Suppl.69):3-17.

10. Delrosso G, Bornacina C, Farinelli P, Bellinzona F, Leigheb G, Colombo E. Bath PUVA and psoriasis: Is a milder treatment a worse treatment? Dermatology (Basel). 2008;216:191-3.

11. Zanolli M. Phototherapy treatment of psoriasis today. J Am Acad Dermatol. 2003;49(Suppl.2):S78-86.

12. Feldman SR, Koo JY, Lebwohl MG, Menter A, van Voorhees A. The psoriasis and psoriatic arthritis pocket guide: Treatment algorithms and management options. Third edition. The National Psoriasis Foundation. New York, 2009. pag 84-91

13. Lim HW, Hönigsmann H, Hawk JLM. Photodermatology. Informa healthcare USA, New York, 2007. pag 319-335.

14. Hönigsmann H. Phototherapy for psoriasis. Clin Exp Dermatol. 2001;26:343-50
15. DeSilva B, McKenzie RC, Hunter JAA, Norval M. Local effects of TL01 phototherapy in psoriasis. Photodermatol Photoimmunol Photomed. 2008;24:268-9.

16. Gläser R, Navid F, Schuller W, Jantschitsch C, Harder J, Schröder $\mathrm{JM}$, et al. UV-B radiation induces the expression of antimicrobial peptides in human keratinocytes in vitro and in vivo. J Allergy Clin Immunol. 2009;123:1117-23.

17. Yoshimura M, Namura S, Akamatsu H, Horio T. Antimicrobial effects of phototherapy and photochemotherapy in vivo and in vitro. Br J Dermatol. 1996;134:528-32.

18. Parrish JA, Jaenicke KF. Action spectrum for phototherapy of psoriasis. J Invest Dermatol. 1981;76:359-62.

19. Storbeck K, Hölzle E, Schürer N, Lehmann P, Plewig G. Narrowband UVB (311 nm) versus conventional broad-band UVB with and without dithranol in phototherapy for psoriasis. J Am Acad Dermatol. 1993;28(Pt.1):227-31.

20. Walters IB, Burack LH, Coven TR, Gilleaudeau P, Krueger JG. Suberythemogenic narrow-band UVB is markedly more effective than conventional UVB in treatment of psoriasis vulgaris. J Am Acad Dermatol. 1999;40(Pt.1):893-900.

21. Coven TR, Burack LH, Gilleaudeau R, Keogh M, Ozawa M, Krueger JG. Narrowband UV-B produces superior clinical and histopathological resolution of moderate-to-severe psoriasis in patients compared with broadband UV-B. Arch Dermatol. 1997;133:1514-22.

22. Yones SS, Palmer RA, Garibaldinos TT, Hawk JLM. Randomized double-blind trial of the treatment of chronic plaque psoriasis: Efficacy of psoralen-UV-A therapy Vs. narrowband UV-B therapy. Arch Dermatol. 2006;142:836-42.

23. Markham T, Rogers S, Collins P. Narrowband UV-B (TL-01) phototherapy Vs. oral 8-methoxypsoralen psoralen-UV-A for the treatment of chronic plaque psoriasis. Arch Dermatol. 2003;139:325-8.

24. Gordon PM, Diffey BL, Matthews JN, Farr PM. A randomized comparison of narrow-band TL-01 phototherapy and PUVA photochemotherapy for psoriasis. J Am Acad Dermatol. 1999;41(Pt.1):728-32.

25. Jury CS, McHenry P, Burden AD, Lever R, Bilsland D. Narrowband ultraviolet B (UVB) phototherapy in children. Clin Exp Dermatol. 2006;31:196-9.

26. Pasic A, Ceovic R, Lipozencic J, Husar K, Susic SM, Skerlev M, et al. Phototherapy in pediatric patients. Pediatr Dermatol. 2003;20:71-7.

27. Valkova S. UVB phototherapeutic modalities. Comparison of two treatments for chronic plaque psoriasis. Acta Dermatovenerol Alp Panonica Adriat. 2007;16:26-30.

28. Kleinpenning MM, Smits T, Boezeman J, van de Kerkhof PCM, Evers AWM, Gerritsen MJP. Narrowband ultraviolet B therapy in psoriasis: Randomized double-blind comparison of high-dose and low-dose irradiation regimens. Br J Dermatol. 2009;161:1351-6.

29. Boztepe G, Akinci H, Sahin S, Karaduman A, Evans SE, Erkin G, et al. In search of an optimum dose escalation for narrowband UVB phototherapy: Is it time to quit 20\% increments? J Am Acad Dermatol. 2006;55:269-71.

30. Ibbotson SH, Bilsland D, Cox NH, Dawe RS, Diffey B, Edwards C, et al. An update and guidance on narrowband ultraviolet B phototherapy: A British Photodermatology Group Workshop Report. Br J Dermatol. 2004;151:283-97.

31. Wainwright NJ, Dawe RS, Ferguson J. Narrowband ultraviolet B (TL-01) phototherapy for psoriasis: Which incremental regimen? Br J Dermatol. 1998;139:410-4. 
32. Olson RL, Sayre RM, Everett MA. Effect of anatomic location and time on ultraviolet erythema. Arch Dermatol. 1966;93:211-5.

33. Schneider LA, Hinrichs R, Scharffetter-Kochanek K. Phototherapy and photochemotherapy. Clin Dermatol. 2008;26:464-76.

34. Karrer S, Eholzer C, Ackermann G, Landthaler M, Szeimies RM. Phototherapy of psoriasis: Comparative experience of different phototherapeutic approaches. Dermatology (Basel). 2001;202:108-15.

35. Zanolli, MD, Feldman SR. Phototherapy treatment protocols: For psoriasis an other phtotherapy responsive dermatoses. New York: Taylor and Francis Group; 2004.

36. Calle J, Rivas AM, Ruiz MC, Sánchez MA, Trujillo MC, Jiménez SB, et al. Manejo de la psoriasis con luz ultravioleta A más psoraleno (PUVA) y luz ultravioleta B (UVB). Rev Asoc Colomb Dermatol. 2009;17:162-71.

37. Haddican MM, Bhutani T, McClelland PB, Koo JYM. Why are there significant differences in published narrowband ultraviolet B dosimetry recommendations? The need for national standardization of phototherapy treatment. J Am Acad Dermatol. 2011;65:411-4.

38. Cameron H, Dawe RS, Yule S, Murphy J, Ibbotson SH, Ferguson J. A randomized, observer-blinded trial of twice Vs. three times weekly narrowband ultraviolet $B$ phototherapy for chronic plaque psoriasis. Br J Dermatol. 2002;147:973-8.

39. Dawe RS, Wainwright NJ, Cameron H, Ferguson J. Narrow-band (TL-01) ultraviolet B phototherapy for chronic plaque psoriasis: Three times or five times weekly treatment? Br J Dermatol. 1998;138:833-9.

40. Boztepe G, Karaduman A, Sahin S, Hayran M, Kölemen F. The effect of maintenance narrow-band ultraviolet B therapy on the duration of remission for psoriasis: A prospective randomized clinical trial. Int J Dermatol. 2006;45:245-50.

41. Stern RS, Armstrong RB, Anderson TF, Bickers DR, Lowe NJ, Harber L, et al. Effect of continued ultraviolet B phototherapy on the duration of remission of psoriasis: A randomized study. J Am Acad Dermatol. 1986;15:546-52.

42. Kragballe K. Vitamin D and UVB radiation therapy. Cutis. 2002;70(Suppl.5):9-12.

43. Guenther LC. Optimizing treatment with topical tazarotene. Am J Clin Dermatol. 2003;4:197-202.

44. Koo JY, Lowe NJ, Lew-Kaya DA, Vasilopoulos AI, Lue JC, Sefton $\mathrm{J}$, et al. Tazarotene plus UVB phototherapy in the treatment of psoriasis. J Am Acad Dermatol. 2000;43(Pt.1):821-8.

45. Meola T Jr, Soter NA, Lim HW. Are topical corticosteroids useful adjunctive therapy for the treatment of psoriasis with ultraviolet radiation? A review of the literature. Arch Dermatol. 1991;127:1708-13.

46. Morison WL, Parrish JA, Fitzpatrick TB. Controlled study of PUVA and adjunctive topical therapy in the management of psoriasis. $\mathrm{Br}$ J Dermatol. 1978;98:125-32.
47. Schmoll M, Henseler T, Christophers E. Evaluation of PUVA, topical corticosteroids and the combination of both in the treatment of psoriasis. Br J Dermatol. 1978;99:693-702.

48. Marcil I, Stern RS. Squamous-cell cancer of the skin in patients given PUVA and ciclosporin: Nested cohort crossover study. Lancet. 2001;358:1042-5.

49. Kaya TI, Tiftik N, Tursen U, Ikizoglu G, Yalcin A. Ultraviolet recall phenomenon associated with methotrexate and cytarabine. J Eur Acad Dermatol Venereol. 2006;20:353-4.

50. Paul BS, Momtaz K, Stern RS, Arndt KA, Parrish JA. Combined methotrexate--ultraviolet $B$ therapy in the treatment of psoriasis. J Am Acad Dermatol. 1982;7:758-62.

51. Lebwohl M, Drake L, Menter A, Koo J, Gottlieb AB, Zanolli M, et al. Consensus conference: Acitretin in combination with UVB or PUVA in the treatment of psoriasis. J Am Acad Dermatol. 2001;45:544-53.

52. Kircik L, Bagel J, Korman N, Menter A, Elmets CA, Koo J, et al. Utilization of narrow-band ultraviolet light B therapy and etanercept for the treatment of psoriasis (UNITE): Efficacy, safety, and patient-reported outcomes. J Drugs Dermatol. 2008;7:245-53.

53. Lucas A, Belinchón I, Pérez-Crespo M, Mataix J, Betlloch I. Successful response to narrow-band UVB in a patient undergoing concomitant treatment with adalimumab for psoriasis. Australas J Dermatol. 2008;49:173-4.

54. Legat FJ, Hofer A, Wackernagel A, Salmhofer W, Quehenberger F, Kerl H, et al. Narrowband UV-B phototherapy, alefacept, and clearance of psoriasis. Arch Dermatol. 2007;143:1016-22.

55. Ibbotson SH, Bilsland D, Cox NH, Dawe RS, Diffey B, Edwards C, et al. An update and guidance on narrowband ultraviolet B phototherapy: A British Photodermatology Group Workshop Report. Br J Dermatol. 2004;151:283-97.

56. Calzavara-Pinton PG, Zane C, Candiago E, Facchetti F. Blisters on psoriatic lesions treated with TL-01 lamps. Dermatology (Basel). 2000;200:115-9.

57. Wolff K, Goldsmith LA, Katz SI, Gilchrest BA, Baller AS, Leffell DJ. Fitzpatrick's Dermatology in General Medicine. Seventh edition, Volume two. McGraw Hill, New York, 2008. pag 2243-2262

58. Lee E, Koo J, Berger T. UVB phototherapy and skin cancer risk: A review of the literature. Int J Dermatol. 2005;44:355-60.

59. Diffey BL. Factors affecting the choice of a ceiling on the number of exposures with TLo1 ultraviolet B phototherapy. Br J Dermatol. 2003;149:428-30.

6o. Stern RS. The risk of squamous cell and basal cell cancer associated with psoralen and ultraviolet A therapy: A 30-year prospective study. J Am Acad Dermatol. 2012;66:553-62.

61. Mudigonda T, Dabade TS, Feldman SR. A review of targeted ultraviolet B phototherapy for psoriasis. J Am Acad Dermatol. 2012;66:664-72. 